\title{
IGLOSS: ITERATIVE GAPLESS LOCAL SIMILARITY SEARCH
}

\author{
BRASLAV RABAR ${ }^{1}$, STRAHIL RISTOV ${ }^{2}$, MAJA ZAGORŠČAK ${ }^{3}$, MARTIN ROSENZWEIG $^{1}$, \\ PAVLE GOLDSTEIN ${ }^{1}$
}

\begin{abstract}
Searching for local sequence patterns is one of the basic tasks in bioinformatics. Sequence patterns might have structural, functional or some other relevance, and numerous methods have been developed to detect and analyze them. These methods often depend on the wealth of information already collected. The explosion in the number of newly available sequences calls for novel methods to explore local sequence similarity. We have developed a high sensitivity web-based iterative local similarity scanner, that finds sequence patterns similar to a submitted query.

Availability: The IGLOSS web server is available at http://compbioserv.math.hr/igloss/
\end{abstract}

\section{INTRODUCTION}

Motif scanning methods are at the heart of many bioinformatics procedures. For example, secondary structure recognition, proteome annotation [Zhang(2003)] and, in general, protein family assignment Bat(2004)], all depend - to a certain extent - on detecting a variant of a (amino acid) motif in a given sequence or a set of sequences. Consequently, numerous methods - Smith-Waterman algorithm Waterman(1976)], BLAST Alt(1990), PSSM Gribskov(1987)], Viterbi Viterbi(1967)] - and applications - e.g. FIMO Grant(2011) have been developed. Typically, the application takes a motif profile as an input, and then, using a dynamic programming approach, or some approximation, finds a version of optimal local alignment in each scanned sequence. Clearly, accuracy of this approach depends - among other things - on the motif profile being of a sufficient quality and size. A considerable increase in the number of newly available sequences, where only a small portion has been properly analysed, makes the task of creating an unbiased, representative profile increasingly difficult, and necessitates a different approach.

In this note, we present IGLOSS - iterative gapless local similarity search - a web-application that will, in a proteome or a collection of proteomes, find sequence patterns similar to a submitted query. The query can consist of one or more sequences of equal length, the level of required similarity can be easily controlled, and we provide simple options for conserved/neutral positions, as well.

\section{IMPLEMENTATION}

Our iterative approach is implemented in a straightforward fashion: initially, a crude profile - with crudeness depending on the size of the query - is built, and the dataset is scanned, with the maximal log-odds score reported for each sequence. Standard results (see de Haan and Ferreira(2006)] guarantee that the scores will be approximately logistically distributed, and motifs with scores above the predetermined scale are used to build a new profile. Clearly, this procedure stops when there is no change in the list of positives, or the predetermined number of iterations is reached.

Let us assume that we are given a motif $M$, of length $k$. To maximize log-odds scores, we compute, for each sequence $x$, the log-odds vector $v(x)$. The components of $v(x)=\left(v(x)_{1}, v(x)_{2}, \ldots, v(x)_{n-k+1}\right)$ are given by

$$
v(x)_{l}=\sum_{i=0}^{k-1} \log \frac{P\left(x_{l+i} \mid y_{i+1}\right)}{P\left(x_{l+i} \mid q\right)}, \quad l=1, \ldots, n-k+1
$$

where $\left\{y_{1}, \ldots, y_{k}\right\}$ and $q$ are distributions determining the position specific scoring matrix for $M$ (in other words, a motif profile). The vector $v(x)$ is computed using a modification of the fast indexed string matching 
algorithm from Ristov(2016)], which considerably reduces overall processing time. Distributions $\left\{y_{1}, \ldots, y_{k}\right\}$ - or, rather, $\left\{y_{1}^{(j)}, \ldots, y_{k}^{(j)}\right\}$ - represent emission probabilities for each column of $M$ in $j$-th iteration, while $q$ is the background distribution for the standard amino acid alphabet. Clearly, $\left\{y_{1}^{(j)}, \ldots, y_{k}^{(j)}\right\}$ - and the way they are refined through iterations - are among the essential aspects of the iteration process. We compute these distributions from the list of positives from the previous iteration - or just the query, for the first iteration. We use a mild sequence-weighting scheme (from Henikoff(1994)], and, depending on the query size, add evolutionary pseudo-counts, given by a high power of the PAM matrix Dayhoff(1987)]. Finally, we average this with the initial distributions - denoted by $\left\{y_{1}^{(1)}, \ldots, y_{k}^{(1)}\right\}$ - in order to prevent divergence. We give a detailed description of this procedure on the server website.

\section{Example And Evaluation}

We applied our server to the GDSL-lipase protein family from four higher plants - Arabidopsis thaliana (AT) (TAIR9), Oryza sativa (OS) (MSU v7), Solanum tuberosum (ST) (ITAG1), and Solanum lycopersicum (SL) (ITAG2.3). Proteins in this family display fairly low overall sequence similarity, but are reasonably well described by the presence of five characteristic motifs (also called blocks), with blocks I, III and V being more conserved ([Chep(2012)], Vujaklija(2016)] $)$. The evaluation consisted of submitting a single sequence, typical for block I, to our scanner, and checking the annotation of sequences in which positive hits are found. We measure the quality of our results by computing true positive rate (TPR), i.e. sensitivity, and positive predictive value (PPV), i.e. precision, and compare them to those obtained by iterative BLAST (i.e. PSIBLAST, Alt(1997)]). PSI-BLAST was used with the same input and with the standard parameters, and with e-value adjusted to obtain approximately the same number of positives. Annotation was determined by further processing the information from GoMapMan Ramšak(2014) resource. The sizes of the resulting protein families (per organism) are given in the central column of Table 1. All data sets - proteomes, lists of positives, as well as queries and a detailed description of the processing procedure - are available from the server website.

\begin{tabular}{|c|c|c|c|c|c|c|c|c|c|c|c|}
\hline Organism & $\mathrm{P}$ & $\mathrm{TP}$ & PPV & TPR & scale & condition positive & blast $P$ & blast TP & blast PPV & blast TPR & evalue \\
\hline $\mathrm{AT}$ & 106 & 85 & 0.80 & 0.72 & 8 & $118(104)$ & 106 & 75 & 0.71 & 0.64 & 480 \\
\hline OS & 114 & 108 & 0.95 & 0.70 & 10 & $155(116)$ & 115 & 86 & 0.75 & 0.55 & 900 \\
\hline $\mathrm{ST}$ & 124 & 89 & 0.72 & 0.72 & 7.5 & $123(123)$ & 123 & 76 & 0.62 & 0.62 & 600 \\
\hline $\mathrm{SL}$ & 108 & 89 & 0.82 & 0.82 & 8 & $108(108)$ & 107 & 76 & 0.71 & 0.70 & 470 \\
\hline
\end{tabular}

TABLE 1. Results for GDSL-like motif query FVFGDSLSDA. The results for IGLOSS are presented on the left and the results for PSI-BLAST on the right. Labels: P - positive, TP - true positive, $\mathrm{CP}$ - condition positive, $\mathrm{PPV}=\mathrm{TP} / \mathrm{P}, \mathrm{TPR}=\mathrm{TP} / \mathrm{CP}$; in brackets is the size of the family (per organism), with isoforms counted once

As can be seen from Table 1, our application is more accurate than PSI-BLAST, with our PPV and TPR consistently higher. While PSI-BLAST is a more versatile and much faster application - GDSL-lipase search on a rice proteome takes around 60 seconds with IGLOSS, and a couple of seconds with PSI-BLAST - this decrease in speed is accompanied by a significant increase in accuracy. Another option for a comparison would be the HMMer-suite, and its iterative module [Finn(2015)], but we selected PSI-BLAST, primarily for the ease of use. It should also be pointed out that a comparison with non-iterative methods would be unfair. The mathematical background of our method is very similar to that of BLAST, with main differences in implementation being purpose-specific model building and the distribution parameter estimation. While 
this is certainly time consuming - especially the latter - it appears that it contributes towards considerably greater accuracy.

In conclusion, we suggest that our method is a viable alternative when it comes to motif scanning, protein family analysis, and even proteome annotation. On the other hand, while IGLOSS can be used as a fast iterative motif scanner, its primary aim is to help researchers explore common sequence patterns in a proteome.

\section{FUNDING}

This work has been partially supported by the European Regional Development Fund [KK.01.1.1.01.0009 - DATACROSS] (SR), the Slovenian Research Agency [J7-7303] (MZ) and the Croatian Science Foundation [IP-2014-09-2285] (BR) and [IP-2016-06-1046] (PG).

\section{REFERENCES}

[Alt(1990)] Altschul, S. F., Gish, W., Miller, W., Myers, E. W., and Lipman, D. J. (1990). Basic local alignment search tool. Journal of molecular biology, 215(3), 403-10.

[Alt(1997)] Altschul, S. F., Madden, T. L., Schäffer, A. A., Zhang, J., Zhang, Z., Miller, W., and Lipman, D. J. (1997). Gapped BLAST and PSI-BLAST:a new generation of protein database search programs. Nucleic Acids Research, 25(17), 3389-3402. [Bat(2004)] Bateman, A., Coin, L., Durbin, R., Finn, R. D., Hollich, V., Griffiths-Jones, S., Khanna, A., Marshall, M., Moxon, S., Sonnhammer, E. L. L., Studholme, D. J., Yeats, C., and Eddy, S. R. (2004). The pfam protein families database. Nucleic Acids Research, 32(SI), D138-D141.

[Chep(2012)] Chepyshko, H., Lai, C. P., Huang, L. M., Liu, J. H., and Shaw, J. F. (2012). Multifunctionality and diversity of GDSL esterase/lipase gene family in rice (Oryza sativa L. japonica) genome: New insights from bioinformatics analysis. $B M C$ Genomics, 13(1).

[Dayhoff(1987)] Dayhoff, M. and Schwartz, R. (1978). A Model of Evolutionary Change in Proteins. In Atlas of protein sequence and structure, pages $345-352$.

[de Haan and Ferreira(2006)] de Haan, L. and Ferreira, A. (2006). Extreme Value Theory: An Introduction. Springer

[Finn(2015)] Finn, R. D., Clements, J., Arndt, W., Miller, B. L., Wheeler, T. J., Schreiber, F., Bateman, A., and Eddy, S. R. (2015). Hmmer: web server 2015 update. Nucleic Acids Research, 43(W1), W30-W38.

[Grant(2011)] Grant, C. E., Bailey, T. L., and Noble, W. S. (2011). FIMO: Scanning for occurrences of a given motif. Bioinformatics, $\mathbf{2 7}(7)$, 1017-1018.

[Gribskov(1987)] Gribskov, M., McLachlan, A., and Eisenberg, D. (1987). Profile analysis: detection of distantly related proteins. Proceedings of the National Academy of Sciences of the United States of America, 84(13), 4355-4358.

[Henikoff(1994)] Henikoff, S. and G. Henikoff, J. (1994). Position-Based Sequence Weights. Journal of molecular biology, 243, $574-578$.

[Ramšak(2014)] Ramšak, Ž., Baebler, Š., Rotter, A., Korbar, M., Mozetič, I., Usadel, B., and Gruden, K. (2014). Gomapman: Integration, consolidation and visualization of plant gene annotations within the mapman ontology. Nucleic Acids Research, 42(D1), 1167-1175.

[Ristov(2016)] Ristov, S. (2016). A fast and simple pattern matching with hamming distance on large alphabets. Journal of Computational Biology, 23(11), 874-876.

[Viterbi(1967)] Viterbi, A. J. (1967). Error Bounds for Convolutional Codes and an Asymptotically Optimum Decoding Algorithm. IEEE Transactions on Information Theory, 13(2), 260-269.

[Vujaklija(2016)] Vujaklija, I., Bielen, A., Paradžik, T., Bidin, S., Goldstein, P., and Vujaklija, D. (2016). An effective approach for annotation of protein families with low sequence similarity and conserved motifs: Identifying GDSL hydrolases across the plant kingdom. BMC Bioinformatics, 17(1), 1-17.

[Waterman(1976)] Waterman, M., Smith, T., and Beyer, W. (1976). Some biological sequence metrics 1. Advances in Mathematics, 20(3), 367-387.

[Zhang(2003)] Zhang, Y., Yin, Y., Chen, Y., Gao, G., Yu, P., Luo, J., and Jiang, Y. (2003). PCAS - A precomputed proteome annotation database resource. BMC Genomics, 4, 1-7.

\footnotetext{
${ }^{1}$ Mathematics Department, Faculty of Natural Sciences and Mathematics, BijeničKa 30, 10000 Zagreb, Croatia ${ }^{2}$ RuĐer Bošković Institute, Bijenička 54, 10000 Zagreb, Croatia ${ }^{3}$ Department of Biotechnology and Systems Biology, National Institute of Biology, VeČna pot 111, 1000 Ljubljana, Slovenia

E-mail address: pavle.goldstein@math.hr
} 